\title{
Advanced Cognitive-Behavioral Treatment Model with Exposure- Response Prevention for Treating Obsessive-Compulsive Disorder
}

\author{
Ju-Wan Kim, Hee-Ju Kang, Ju-Yeon Lee, Sung-Wan Kim, II-Seon Shin, and Jae-Min Kim ${ }^{凶}$ \\ Department of Psychiatry, Chonnam National University Medical School, Gwangju, Republic of Korea
}

\section{INTRODUCTION}

Obsessive-compulsive disorder (OCD) is characterized by recurrent obsessions and compulsions, which are significantly associated with psychic distress and functional impairment. ${ }^{1}$ Meanwhile the treatment modality and clinical outcomes for patients with OCD are also highly complex leading to decimating clinical course in naturalistic treatment setting. ${ }^{2,3}$ Exposureresponse prevention (ERP) therapy entails learning how to reduce fear (habituation) without engaging in the compulsive behavior after experiencing the anxiety-producing stimulus [conditioned stimuli (CS)]. Foa et al. discussed habituation based on emotional processing theory (EPT) and argued that between-session habituation was the most important treatment. ${ }^{4-6}$ Although ERP therapy is effective for many patients with OCD, not all patients respond to the treatment, as $25-50 \%$ of patients discontinue the therapy or experience recurrence. Some researchers have argued that habituation, which is generally understood to be the most important aspect of ERP therapy, may not be the key for treatment. ${ }^{8}$ Craske et al. proposed inhibitory learning theory (ILT) as an alternative to EPT. ${ }^{8-10}$ Based on fear extinction research, they proposed a framework for exposure in which inhibitory learning is optimized to enhance long-term outcomes. In fact, both EPT and ILT use learning theory (i.e., classical and operant conditioning) to understand and treat patients. This article examines limitations of applying learning theory (Supplementary Materials in the online-only Data Supplement) that we identified during ERP

\footnotetext{
Received: September 6, 2020 Revised: September 14, 2020

Accepted: September 15, 2020

$\triangle$ Correspondence: Jae-Min Kim, MD, PhD

Department of Psychiatry, Chonnam National University Medical School, 160 Baekseo-ro, Dong-gu, Gwangju 61469, Republic of Korea

Tel: +82-62-220-6143, Fax: +82-62-225-2351

E-mail: jmkim@chonnam.ac.kr

(c) This is an Open Access article distributed under the terms of the Creative Commons Attribution Non-Commercial License (https://creativecommons.org/licenses/by$\mathrm{nc} / 4.0$ ) which permits unrestricted non-commercial use, distribution, and reproduction in any medium, provided the original work is properly cited.
}

therapy in patients with OCD. Based on this understanding, we propose an advanced cognitive-behavioral theory (CBT) model of OCD by combining learning theory and Beck's cognitive model to better apply ERP therapy to OCD.

\section{ADVANCED MODEL FOR OCD CBT WITH ERP}

In this article, we propose an advanced CBT model with ERP for treatment of OCD by applying Beck's cognitive model to learning theory. ${ }^{11}$ Beck's cognitive model states that thoughts, emotions, and behaviors are mutually influenced by each other. Shifting cognition is seen as the main mechanism by which lasting emotional and behavioral changes take place. We illustrate the combination of Beck's cognitive model and learning theory in Supplementary Figure 1 (in the online-only Data Supplement).

The newly proposed CBT model for OCD is shown in Figure 1. For this CBT model, we identified four elements of ERP therapy (Figure 1) to explain the difference between ILT and EPT. For easy application in a clinical situation, the terminology used are the words used for OCD in the DSM-5. The reasons for dividing each step into four processes are as follows.

Process A consists of an environmental factor and perception. This step suggests the stimulus that causes the obsession, as described in classical conditioning, and the pathway by which patients receive the external situmulus. For example, external environments (e.g., waste and handles) are important to OCD patients who engage in excessive washing behavior, but they perceive these objects as contaminated, so they become obsessed with them. We named this process perception. People can sense tactically, and the handle of a door may feel different for each person. It may feel smooth to one, while it may feel sticky to another person. In other words, objective facts are received differently by each person, and such perceptions are fundamental to the cognitive model. By using this 


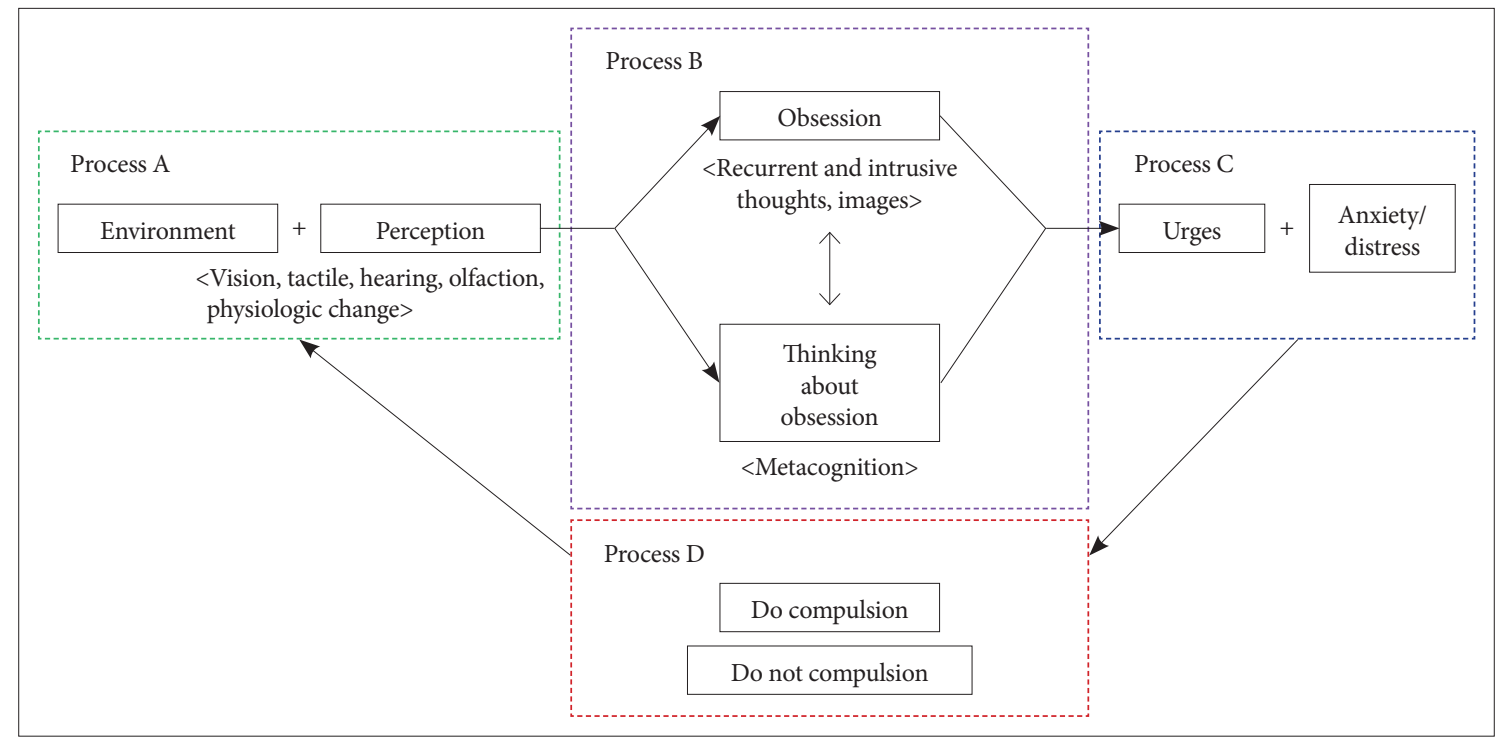

Figure 1. New model for cognitive-behavioral therapy (CBT) with obsessive-compulsive disorder (OCD).

concept, it is possible to account for the greater frequency and longer duration of compulsion, which are not explained by existing learning theory. Patients may wash their hands for 20 minutes or longer after gripping a door handle to remove the stickiness they feel on their hands. However, they may still feel sticky for longer than 20 minutes. Moreover, another environmental stimulus, water may also feel sticky. Hence more compulsive washing may be performed to remove the stickiness. Process A may help to clarify why patients experience obsessions and compulsions. Instead of just identifying the stimuli that cause the obsession and compulsion, a therapist will perform ERP therapy better when they know how the patient is perceiving the stimulus.

Process B consists of obsession and thinking about the obsession. The key at this step is the notion of "thinking about the obsession," i.e., the concept of metacognition. Metacognition, or thinking about thinking, helps people to develop their problem-solving ability by learning what works, and it serves as a core part of self-regulated learning. ${ }^{12,13}$ After process $\mathrm{A}$, the patient experiences an obsession, and their metacognition finds a way to solve this problem of obsession (i.e., they think about their obsession). By presenting this process, we can more easily explain the differences between EPT and ILT in ERP therapy. The exposure therapy on which ILT focuses encourages patients to understand the intention of the therapy and increase their motivation for participating in therapy, educating them about the useful aspects of not acting compulsively, though they experience unpleasant perceptions, obsessions, urges, and anxiety/distress. The patients then go through an ERP session to learn inhibition. After process A, they may understand that they can actively select one of two learning types: 1) excitatory learning, in which they undergo the previous com- pulsion, or 2) inhibitory learning, acquired during therapy. Repeated ERP sessions involve the process of training the patient to choose the latter. At this time, if the patient experiences habituation while doing ERP sessions, the treatment effect may be increased.

Process $\mathrm{C}$ consists of managing urges, anxiety/distress, and habituation, as it is important in ERP therapy to apply EPT at this stage. Process D focuses on the compulsion and not enacting the compulsion. After this step, the patient repeats the steps by returning to process $\mathrm{A}$.

The advantages of applying Beck's cognitive model to learning theory are as follows. First, considering the limitations of the aforementioned learning theory, Beck's cognitive model is more applicable to OCD because it has been used to treat depressive disorder. Furthemore, rumination, which is frequently found in patients with depression, is similar to obsession in OCD. Second, the frequency and duration of the compulsion can be explained. The key to Beck's cognitive therapy is to identify and correct how the patient's inner psyche accepts what has happened. If this is explained based on criterion $\mathrm{B}$ for $\mathrm{OCD}$ in the DSM-5, patients' compulsive behavior, which is intended to relieve anxiety, is inadequate, so they repeatedly and continuously experience the compulsion. Finally, it is useful for describing ERP treatment with ILT. In the proposed model, the application of ERP to ILT focuses on process B and D rather than the habituation experience (process $\mathrm{C}$ ).

We present two cases in which ILT was applied using the above model (Supplementary Materials in the online-only Data Supplement). To show the differences between this and EPT treatment, one case is a treatment case with habituation and inhibitory learning, and the other is a case of inhibitory learning treatment without habituation. 


\section{DISCUSSION}

A recently published article found that difficulty with inhibiting OCD behaviors may result from a more intense initiation of responses..$^{14}$ The authors asserted that OCD patients demonstrated compulsive behavior and impaired inhibitory control under conditions of conflict. ILT can be used to help CBT therapists teach patients to suppress urges during behavioral therapy. ILT suggests that two kinds of learning occur due to the obsession triggered by the CS: 1) excitatory learning acquired during previous fear acquisition (conditioned stimulus-unconditioned stimulus) and 2) new inhibitory learning acquired during ERP (conditioned stimulus-no unconditioned stimulus). ${ }^{8}$ Expectancy violation during exposure helps to establish an inhibitory association with a CS that had previously been associated with an excitatory response. ${ }^{9}$ However, the empirical literature evaluating hypotheses derived from the inhibitory learning approach to exposure is lacking. We have suggested some limitations of learning theory to elucidate existing OCD theory (Supplementary Materials in the online-only Data Supplement), and have provided an advanced CBT model for overcoming such limits.

The most important difference between EPT and ILT is the cognitive approach to obsession and compulsion. The cognitive approach focused on EPT aims to allow patients to learn that their obsessional anxiety is not permanent and that, until habituation is effective, they should resist the urge to perfrom the compulsive behavior to avoid discomfort. ${ }^{15}$ Unfortunately, only a few patients experience habituation during treatment. It is very difficult to force patients who visit a hospital due to distress derived from an obsession to withstand discomfort that is not easily alleviated, which often leads to treatment failure. On the other hand, habituation is not a treatment goal in ILT, but a part of the treatment process. We believe that increasing the patient's tolerance for anxiety, distress, and urges has important clinical value in treating OCD and complements the goal of inhibitory learning. The patient is repeatedly exposed to the CS to learn to not perform the compulsion in response to a given obsession, with or without habituation. In this regard, ERP using ILT is expected to contribute to enhancing patient compliance with the therapy.

When we apply this advanced CBT model, the patients who did not experience habituation but was more confident in controlling themselves through the process $\mathrm{D}$ of 'Do not compulsion.' We focused on the fact that the ILT approach could allow patients to more actively participate in the therapy. Encouraging patients to recognize their ability to actively control obsessions while participating in ERP is very important for the therapy. Olatunji et al. ${ }^{16}$ found that the core of OCD is a negative appraisal of intrusive thoughts, with thoughts such as "I am out of control" and "I am a terrible person." Since these metacognitons were identified after the development of classical and operant learning, learning theory cannot be applied perfectly to them. The term "thinking about obsession" in our CBT model is useful to make up for the shortcomings of these learning theories. When we use this model in a cognitive approach, we focus on patients' thinking about their obsessions, not on patients' obsessions.

However, the present model also has some limitations. It was somewhat difficult to explain mental compulsion using this model. Perhaps the reason for this is that the proposed model involves patient behavior during process $\mathrm{D}$, as mental compulsion is the act of thinking about the obsession (process B) rather than an active behavior itself. These concepts need to be modified or supplemented through further discussion.

The goal of this article was to present an approach to optimizing the inhibitory learning component of ERP. Although we offer an advanced model for facilitating this goal, therapists should exercise some flexibility when applying this model.

\section{Supplementary Materials}

The online-only Data Supplement is available with this article at https://doi.org/10.30773/pi.2020.0340.

\section{Acknowledgments}

This study was supported by a grant (B2020-0208) Chonnam National University Hospital Biomedical Research Institute to J-MK.

\section{Conflicts of Interest}

The authors have no potential conflicts of interest to disclose.

\section{Author Contributions}

Conceptualization: Ju-Wan Kim. Data curation: Ju-Wan Kim. Funding acquisition: Jae-Min Kim. Investigation: Ju-Wan Kim. Methodology: JuWan Kim. Project administration: Sung-Wan Kim, Il-Seon Shin, Jae-Min Kim. Supervision: Jae-Min Kim. Visualization: Ju-Wan Kim, Hee-Ju Kang, Ju-Yeon Lee, Il-Seon Shin. Writing_original draft: Ju-Wan Kim. Writing-review \& editing: Ju-Wan Kim, Sung-Wan Kim, Jae-Min Kim.

\section{ORCID iDs}

Ju-Wan Kim

Hee-Ju Kang

Ju-Yeon Lee

Sung-Wan Kim

Il-Seon Shin

Jae-Min Kim https://orcid.org/0000-0002-9888-1090 https://orcid.org/0000-0001-5113-8841 https://orcid.org/0000-0003-0653-7223 https://orcid.org/0000-0002-6739-2163 https://orcid.org/0000-0001-5370-7649 https://orcid.org/0000-0001-7409-6306

\section{REFERENCES}

1. American Psychiatric Association. Diagnostic and Statistical Manual of Mental Disorders (5th edition). Washington, DC: American Psychiatric Association Publishing; 2013.

2. Garg H, Kumar S, Singh S, Kumar N, Verma R. New onset obsessive compulsive disorder following high frequency repetitive transcranial magnetic stimulation over left dorsolateral prefrontal cortex for treatment of negative symptoms in a patient with schizophrenia. Clin Psychopharmacol Neurosci 2019;17:443-445.

3. Yoon HJ, Seo EH, Kim JJ, Choo IH. Neural correlates of self-referential 
processing and their clinical implications in social anxiety disorder. Clin Psychopharmacol Neurosci 2019;17:12-24.

4. Foa EB, Kozak MJ. Emotional processing of fear: exposure to corrective information. Psychol Bull 1986;99:20-35.

5. Foa EB, McNally RJ. Mechanisms of Change in Exposure Therapy. In: Rapee RM, Editor. Current Controversies in the Anxiety Disorders. New York: Guilford Press; 1996, p.329-343.

6. Foa EB, Huppert JD, Cahill SP. Emotional Processing Theory: An Update. In: Rothbanm BO, Editor. Pathological Anxiety: Emotional Processing in Etiology and Treatment. New York, NY, US: Guilford Press; 2006, p.3-24.

7. Franklin ME, Foa EB. Cognitive-Behavioral Treatments for ObsessiveCompulsive Disorder. In: Gorman JM, Editor. A Guide to Treatments that Work. New York, NY: Oxford University Press; 1998, p.339-357.

8. Craske MG, Kircanski K, Zelikowsky M, Mystkowski J, Chowdhury N, Baker A. Optimizing inhibitory learning during exposure therapy. Behav Res Ther 2008;46:5-27.

9. Craske MG, Liao B, Brown L, Verviliet B. Role of inhibition in exposure therapy. J Exp Psychopathol 2012;3:322-345.

10. Jacoby RJ, Abramowitz JS. Inhibitory learning approaches to exposure therapy: a critical review and translation to obsessive-compulsive disorder. Clin Psychol Rev 2016;49:28-40.

11. Beck AT. Thinking and depression: theory and therapy. Arch Gen Psychiatry 1964;10:561-571.

12. Flavell JH. Metacognition and cognitive monitoring: a new area of cognitive developmental inquiry. Psychology 1979;34:906-911.

13. Folkman S, Moskowitz JT. Coping: pitfalls and promise. Annu Rev Psychol 2004;55:745-774.

14. Dayan-Riva A, Berger A, Anholt GE. Affordances, response conflict, and enhanced-action tendencies in obsessive-compulsive disorder: an ERP study. Psychol Med 2020;7:1-16.

15. Abramowitz JS. Obsessive-compulsive disorder. Lancet 2009;374:491499.

16. Olatunji BO, Christian C, Brosof L, Tolin DF, Levinson CA. What is at the core of $\mathrm{OCD}$ ? A network analysis of selected obsessive-compulsive symptoms and beliefs. J Affect Disord 2019;257:45-54. 


\section{SUPPLEMENTARY MATERIAL}

Kim JW, et al. Advanced cognitive-behavioral treatment model with exposure-response prevention for treating obsessivecompulsive disorder

This supplementary material has been provided by the authors to give readers additional information about their work.

\section{OCD and learning theory}

The most supported neural and pathophysiological model of OCD focuses on overactivation of the cortico-striato-thalamocortical circuits. ${ }^{1}$ According to this model, the orbitofrontal-subcortical pathway is hyperactive in OCD patients compared to healthy people, resulting in a change in the reinforcement contingencies of stimuli. Due to this change in reinforcement contingency, when OCD patients suffer from an obsession, they engage in some kind of behavior that reduces the anxiety or distress. Finally, they come to believe that this behavior is the solution to the obsession, and this learned behavior eventually becomes a compulsion.

\section{Obsession and classical conditioning}

$\mathrm{CBT}$ is a time-limited, structured, and active psychological treatment, and ERP is its main form of application in cases of OCD. ${ }^{2}$ ERP treatment for OCD begins with understanding the formation of the obsession and compulsion by applying learning theory (classical conditioning and operant conditioning) $)^{3-5}$ Classical conditiong theory suggests that, in OCD patients, a neutral stimulus can be overgeneralized and become a CS that evokes obsession. ${ }^{4,6}$ For example, a patient who has an obsession with contamination fears contamination by stimuli that healthy people would not think are dirty. Understanding classical conditioning while performing the therapy is helpful when planning ERP therapy. As a form of directive psychotherapy, ERP can be more effective if the therapist understands the factors (who, when, where, what, and how) that cause the obsession and can therefore clarify all aspects of the obsessive pattern, i.e., the CS, unconditioned stimulus (US), unconditioned response (UR), and conditioned response (CR).

\section{Compulsion and operant conditioning}

The formation of a compulsion can be explained by the theory of operant conditioning. ${ }^{5}$ The Skinner box became an important tool for studying learned behavior and contributed a great deal to our understanding of the effects of reinforcement and punishment. OCD patients judge that a ritual behavior relieves the anxiety or distress caused by an obsession. This belief leads them to engage in the behavior, which is then reinforced, increasing the likelihood that the compulsive behavior will occur again when the obsession recurs. ${ }^{1}$ For example, patients repeatedly wash their hands to reduce anxiety whenever they think their hands are dirty or contaminated. Operant conditioning is also applied to understand the mechanism of habituation when carrying out ERP treatment. ERP approaches are habituation-based models that emphasize reduction in fear through the exposure and response-prevention process as essential for reducing symptoms.

\section{Limitation of learning theory}

Classical and operant conditioning help to explain how OC symptoms are formed and how they can be treated, but there are some limitations. First, both theories have used animal models to explain principles that are then applied to human behavior, including that of psychiatric patients. OCD is a major disease of thoughts and behaviors. Thus, it is difficult to use animal models to fully describe the cognitive aspect of the disease. Second, the theories do not fully explain OCD. Classical conditioning explains how a neutral stimulus causes the obsession, but it does not explain formation of the compulsion. More specifically, in classical conditioning, the response takes the form of a reflex. For example, if a dog salivates after seeing food and/or hearing the sound of a bell, the saliva is not controlled by the dog, but is automatically secreted. However, compulsion is not a reflexive response but a behavior selected (intended) spontaneously by the OCD patient to avoid anxiety or distress deriving from the obsession (motivation). In conclusion, classical conditioning theory alone does not explain how patients learn compulsive behaviors. In contrast, operant conditioning explains the formation of a compulsive behavior relatively well, but it does not explain the association between the obsession and the CS. Finally, the two theories cannot explain the definition contained in criterion B of the Diagnostic and Statistical Manual of Mental Disorders, Fifth Edition (DSM-5): "The obsessions and compulsions are time-consuming (e.g., take more than 1 hour per day)." In other words, they cannot elucidate the frequency or duration of the patient's compulsive behavior. Although patients exhibit a compulsion to relieve the anxiety caused by the obsession when neutral stimuli are applied, it is impossible, using these two theo- 
ries, to explain why they repeatedly exhibit compulsive behavior. For example, when a patient obsessively washes their hands due to a contamination obsession, they should stop washing them and perform other tasks if the hands are clearly washed. Compared to normal subjects, who wash their hands for 1 minute, OCD patients show compulsive washing behavior, several times or for much longer periods. Thus, they cannot stop continuous washing behavior, even after the stimuli that triggered the obsession are removed.

\section{Case}

\section{Contamination and washing case}

Mr. A, a 48-year-old entrepreneur, was seen at another hospital for ERP treatment. He had an obsession with contamination that lasted 4-5 hours/day, and he took 10-30 minutes to wash each hand to relieve the compulsion. He was upset that he could not stop washing and was unable to control his symptoms after 20 minutes of washing his hands. Although he had suffered from this obsession since he was in elementary school, he had not received any therapy. When he was 41 (7 years ago), he presented at another hospital, where he was prescribed medication, as recommended by his wife. He was prescribed $20 \mathrm{mg}$ escitalopram and $0.5 \mathrm{mg}$ alprazolam, and his symptoms were controlled to the extent that others could not identify them. When the washing behavior reappeared 7 months before he came to our hospital and was uncontrolled, he became angry, struck a mirror with his hand, and broke his wrist. He visited a tertiary care hospital due to the severity of the injury. The doctor adjusted his medication to control his obsession and impulsiveness ( $40 \mathrm{mg}$ escitalopram, $1.5 \mathrm{mg}$ risperidone, $0.5 \mathrm{mg}$ clonazepam, and $0.25 \mathrm{mg}$ alprazolam), but his distress declined by only about $20 \%$. Although he began to take $200 \mathrm{mg}$ sertraline, $5 \mathrm{mg}$ aripiprazole, $25 \mathrm{mg}$ clomipramine, $0.5 \mathrm{mg}$ clonazepam, and $2 \mathrm{mg}$ diazepam when the previous drugs proved ineffective, he was referred for ERP therapy, as his symptoms had not significantly improved. We prepared for ERP therapy while adjusting the dosages of medications over a 3-week period to $300 \mathrm{mg}$ sertraline, $5 \mathrm{mg}$ aripiprazole, $0.5 \mathrm{mg}$ clonazepam, and $50 \mathrm{mg}$ fluvoxamine. During the process of exploring and evaluating the patient's OCD symptoms [Yale-Brown Obsessive-Compulsive scale (YBOCS) score: 34], psychoeducation about OCD and treatments was conducted. In particular, the patient had a history of uncontrolled anger and had hurt his wrist as a result, so education designed to keep him from engaging in compulsive washing was implemented, although his urge and distress were not abated. He made an effort to learn how to control the urges. Three weeks after the initial treatment phase, he said that he had $60 \%$ discomfort compared to baseline, and we began exposure therapy. Four sessions of exposure therapy were conducted. After the first habituation session, he reported that he was motivated to pursue the therapy. After session 2, he said that he should wash his hands if he felt sticky, but that washing only for 3 minutes was sufficient to stop the behavior. One week after session 4 , he was satisfied with the therapy, as his discomfort score after the therapy was about 20, so he discontinued the behavioral therapy (YBOCS score: 6). He now reports that, 6 months later, his symptoms are stably controlled without further ERP therapy, and he now takes $200 \mathrm{mg}$ sertraline and $50 \mathrm{mg}$ fluvoxamine.

\section{Doubt and checking behavior case}

Mr. B, a 20-year-old college student, was referred to another hospital for ERP treatment. He spent more than 2 hours/day checking whether his wallet was lost, and expressed concern about others' watching such behavior. For the past 3 years, he worried that he had dropped his wallet whenever he hit a door or got up from a chair. At first, he just turned his head and checked the chair to verify that he had his wallet, but he gradually extended his ritualistic behavior, as such behavior did not reduce his anxiety. He regarded it as a tic and was distressed, because he could not control it. When he visited a therapist, the ritualistic behavior he performed whenever he sat down and stood up was extended. The order and contents of the ritual were as follows: take a long breath $\rightarrow$ open eyes wide $\rightarrow$ look left and right $\rightarrow$ close eyes $\rightarrow$ take a breath $\rightarrow$ recite the numbers 111-11-1111-12345 $\rightarrow$ clench teeth together $\rightarrow$ shake head. The therapist judged that the ritualistic behavior was a compulsion to reduce the obsession with losing his wallet, and recommended ERP therapy. The therapist began by conducting exploration and education about medication and ERP across three sessions. However, Mr. B did not experience habituation and had a difficult time suppressing his compulsion for 90 minutes. Therefore, the therapist extended the number of exposure sessions to two per week to increase the effects of the ERP therapy. However, Mr. B asked to stop the therapy in the middle of a session because of his distress. In addition, he reported that his interpersonal skills and ability to control stress had decreased and that his compulsive symptoms were exacerbated whenever he got depressed due to stressful life events. The therapist determined that outpatient treatment would not have significant effects and recommended hospital treatment. The patient was hospitalized and received therapy for 26 days. His medications were adjusted ( $200 \mathrm{mg}$ fluvoxamine, $75 \mathrm{mg}$ venlafaxine, $2 \mathrm{mg}$ aripiprazole, $0.25 \mathrm{mg}$ clonazepam, $25 \mathrm{mg}$ trazodone, and $40 \mathrm{mg}$ propranolol), and he underwent more intensive exposure therapy. The therapist repeatedly conducted psychoeducation to help him learn that his behavior was not a tic but a com- 
pulsion that he could suppress. The frequency of the compulsion decreased as he gradually realized that he could control his behavior, making him less concerned about others' watching him, and leading to improvement in his depression. When he was discharged from the hospital, his YBOCS score was 11.

\section{REFERENCES}

1. Pauls DL, Abramovitch A, Rauch SL, Geller DA. Obsessive-compulsive disorder: an integrative genetic and neurobiological perspective. Nat Rev Neurosci 2014;15:410-424.

2. Olatunji BO, Davis ML, Powers MB, Smits JA. Cognitive-behavioral therapy for obsessive-compulsive disorder: a meta-analysis of treatment outcome and moderators. J Psychiatr Res 2013;47:33-41.

3. Barlow DH. Anxiety and Its Disorders: The Nature and Treatment of Anxiety and Panic (2nd Ed.). New York, NY: Guilford Press; 2002.

4. Pavlov IP. Conditional Reflexes. New York: Dover Publications; 1960.

5. Skinner BF. Contingencies of Reinforcement: A Theoretical Analysis. New York, NY: Appleton-Century-Crofts; 1969.

6. Lissek S, Powers AS, McClure EB, Phelps EA, Woldehawariat G, Grillon C, et al. Classical fear conditioning in the anxiety disorders: a meta-analysis. Behav Res Ther 2005;43:1391-1424.

7. American Psychiatric Association. Diagnostic and Statistical Manual of Mental Disorders (5th Edition). Washington, DC: American Psychiatric Press; 2013. 


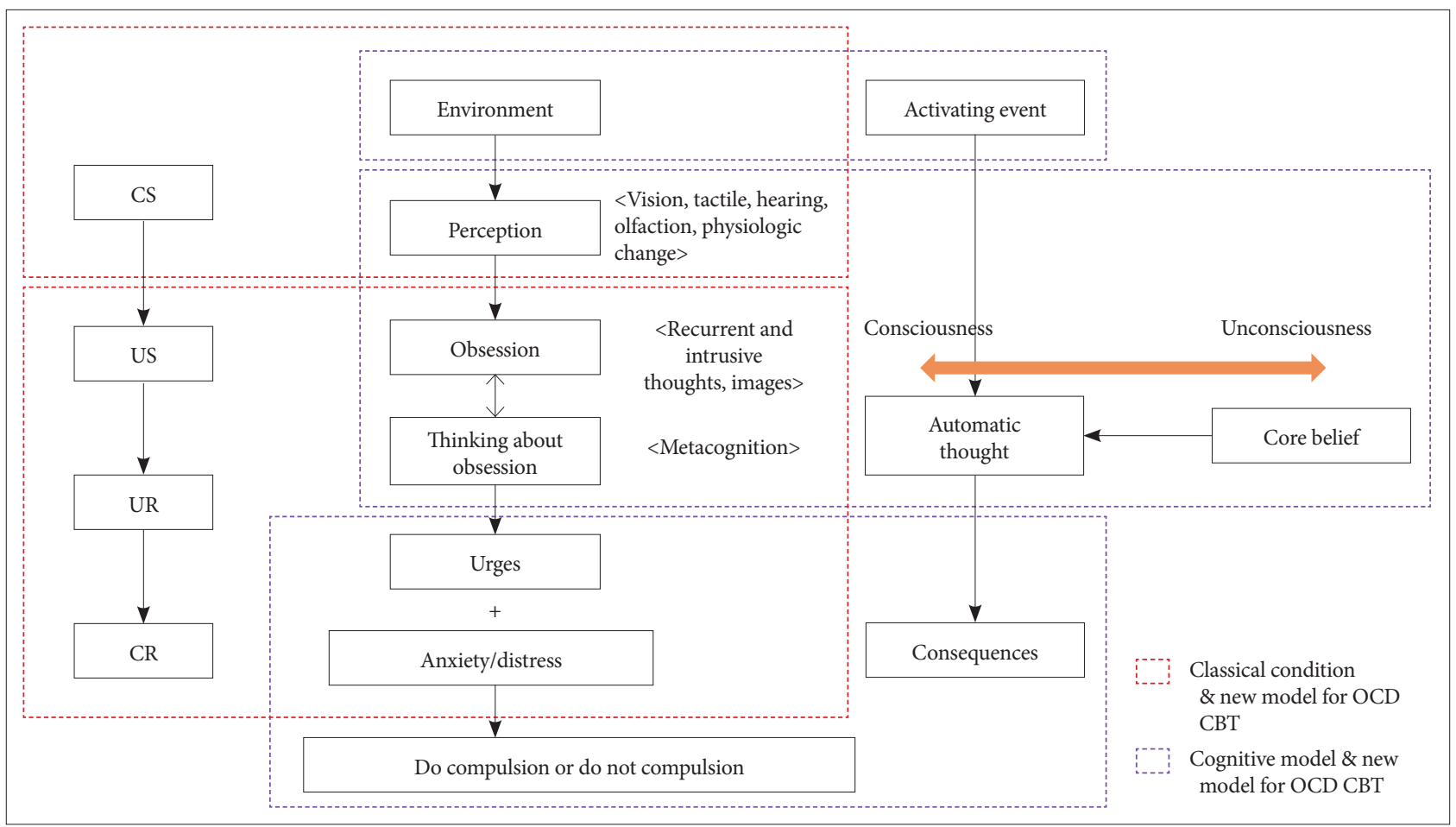

Supplementary Figure 1. Comparison of new and existing perspectives on the cognitive-behavioral therapy (CBT) model for obsessivecompulsive disorder (OCD). CS: conditioned stimulus, US: unconditioned stimulus, UR: unconditioned response, CR: conditioned response. 